\title{
Stereoselective Synthesis of the $\gamma$-Lactam Hydrolysate of the Thiopeptide Cyclothiazomycin
}

\author{
Mark C. Bagley* and Xin Xiong \\ School of Chemistry, Cardiff University, PO Box 912, Cardiff, CF10 3TB, UK. \\ Email Address Bagleymc@cf.ac.uk
}

\section{Materials and Methods}

Commercially available reagents were used as received without further purification; solvents were dried by standard procedures. Light petroleum refers to the fraction with b.p. $40-60{ }^{\circ} \mathrm{C}$ and ether refers to diethyl ether. Unless otherwise stated, reactions were performed under an atmosphere of dry nitrogen. Flash chromatography was carried out using Merck Kieselgel $60 \mathrm{H}$ silica or Matrex silica 60. Analytical thin layer chromatography was carried out using aluminium-backed plates coated with Merck Kieselgel $60 \mathrm{GF}_{254}$ that were visualised under UV light (at 254 and/or $360 \mathrm{~nm}$ ). Preparative thin layer chromatography was carried out using aluminium-backed plates coated with Merck Kieselgel 60 $\mathrm{GF}_{254}$. Visualisation was achieved by UV light (at $254 \mathrm{and} /$ or $360 \mathrm{~nm}$ ) and/or potassium permanganate stain.

Fully characterised compounds were chromatographically homogeneous. Melting points were determined on a Kofler hot stage apparatus. Infra-red spectra were recorded in the range 4000-600 $\mathrm{cm}^{-1}$ on a Perkin-Elmer 1600 series FT-IR spectrometer either as a $\mathrm{KBr}$ disk, nujol mull, in solution in dry dichloromethane or as a thin film between $\mathrm{NaCl}$ plates, as indicated. NMR spectra were recorded using a Bruker DPX 400 instrument operating at $400 \mathrm{MHz}$ for ${ }^{1} \mathrm{H}$ spectra and $100 \mathrm{MHz}$ for ${ }^{13} \mathrm{C}$ spectra (internal standard TMS); $J$ values were recorded in $\mathrm{Hz}$ and multiplicities were expressed by the usual conventions. Low-resolution mass spectra were determined using a Fisons VG Platform II Quadrupole instrument using atmospheric pressure chemical ionisation (APcI) or electron ionisation (EI), as stated. High-resolution mass spectra were obtained courtesy of the EPSRC Mass Spectrometry Service at University College of Wales, Swansea, UK using atmospheric pressure chemical ionization (APcI) or electron ionization (EI). Calculated accurate masses of parent compounds denote the mass of the ion 
(mass of electron is $0.00055 \mathrm{Da}$ ) except where indicated. Microanalyses were recorded using a PerkinElmer 240C Elemental Analyzer in-house, or courtesy of Warick Analytical Services Ltd at University of Warwick Science Park, UK using an Exeter 440 Elemental Analyser. Chiral compounds were analysed using a AA-1000 Polarimeter apparatus from Optical Activity LTD., using the sodium D line and at the indicated temperature, and are given in $\operatorname{deg} \mathrm{cm}^{3} \mathrm{~g}^{-1} \mathrm{dm}^{-1}$ for $[\alpha]$ and $10^{-2} \mathrm{gcm}^{-3}$ for $c$. HPLC was carried out using a HP-1100 HPLC machine equipped with a variable wavelength UV detector and variable flow pump. Microwave experiments were carried out in a CEM Discover ${ }^{\mathrm{TM}}$ microwave synthesiser at the temperature and initial power stated.

\section{(R)-Ethyl 3-amino-4-( $N$-tert-butoxycarbonyl)aminopent-2-enoate (6)}

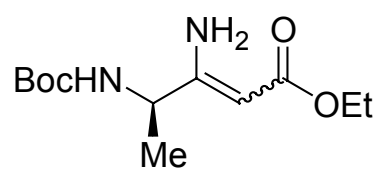

A mixture of (R)-ethyl 4-(N-tert-butoxycarbonyl)amino-3-oxopentanoate (9) ${ }^{1}$ (994 mg, $\left.3.8 \mathrm{mmol}\right)$ and ammonium acetate $(1.47 \mathrm{~g}, 19.0 \mathrm{mmol})$ in ethanol $(30 \mathrm{~mL})$ was stirred at room temperature for $4 \mathrm{~h}$ and concentrated. The residue was partitioned between ethyl acetate $(60 \mathrm{~mL})$ and saturated aqueous sodium hydrogen carbonate solution $(60 \mathrm{~mL})$. The aqueous layer was further extracted with ethyl acetate (60 $\mathrm{mL}$ ) and the organic layers were combined, washed sequentially with saturated aqueous sodium hydrogen carbonate solution $(60 \mathrm{~mL})$, and brine $(60 \mathrm{~mL})$, dried $\left(\mathrm{Na}_{2} \mathrm{SO}_{4}\right)$, evaporated in vacuo, and purified by flash chromatography on silica, eluting with light petroleum-ethyl acetate (7:3), to give the title compound ${ }^{2}(687 \mathrm{mg}, 70 \%)$ as a colourless oil: $[\alpha]_{\mathrm{D}}{ }^{25}+17.0\left(\right.$ c 2.50, $\left.\mathrm{CHCl}_{3}\right)$; IR (KBr) 3458, 3365, 3320, 2983, 2937, 1683, 1612, 1522, 1365, 1310, 1274, 1162, 1042, 861, $792 \mathrm{~cm}^{-1} ;{ }^{1} \mathrm{H}$ NMR (400 $\left.\mathrm{MHz}_{\mathrm{CDCl}}\right) \delta 4.75(1 \mathrm{H}$, br s, NHBoc), $4.54(1 \mathrm{H}, \mathrm{s},=\mathrm{CH}), 4.13(1 \mathrm{H}, \mathrm{q}, J=7.0, \mathrm{CHMe}), 4.04(2 \mathrm{H}$, q, $\left.J=7.1, \mathrm{OCH}_{2} \mathrm{Me}\right), 1.37\left(9 \mathrm{H}, \mathrm{s}, \mathrm{CMe}_{3}\right), 1.28(3 \mathrm{H}, \mathrm{d}, J=7.0, \mathrm{CHMe}), 1.19(3 \mathrm{H}, \mathrm{t}, J=7.1$, $\left.\mathrm{OCH}_{2} \mathrm{Me}\right) ;{ }^{13} \mathrm{C} \mathrm{NMR}\left(100 \mathrm{MHz}, \mathrm{CDCl}_{3}\right) \delta 170.5(\mathrm{C}), 164.7(\mathrm{C}), 155.6(\mathrm{C}), 81.0(\mathrm{CH}), 80.3(\mathrm{C}), 58.7$ $\left(\mathrm{CH}_{2}\right), 49.7(\mathrm{CH}), 28.3(\mathrm{Me}), 19.6(\mathrm{Me}), 14.5(\mathrm{Me})$; MS (APcI) $m / z$ (relative intensity) $259\left(\mathrm{MH}^{+}\right.$, 100\%), 203 (55), 160 (23); HRMS calcd for $\mathrm{C}_{12} \mathrm{H}_{23} \mathrm{~N}_{2} \mathrm{O}_{4}(\mathrm{MH}) 259.1652$, found 259.1648.

\footnotetext{
${ }^{1}$ (a) Honoré, T.; Hjeds, H.; Krogsgaard-Larsen, P.; Christiansen, T. R. Eur. J. Med. Chem. 1978, 13, 429.(b) Kim, H.-O.; Olsen, R. K.; Choi, O.-S. J. Org. Chem. 1987, 52, 4531.

${ }^{2}$ HPLC: ee $=92 \%$; Chiralpak AD, hexane-propan-2-ol (95:5), $\lambda_{\max }=273 \mathrm{~nm}, 1.0 \mathrm{~mL} \mathrm{~min}^{-1}, R_{\mathrm{T}}=15.1 \mathrm{~min}(S$-isomer has $\left.R_{\mathrm{T}}=18.0 \mathrm{~min}\right)$.
} 


\section{2,2-Diethoxythioacetamide}

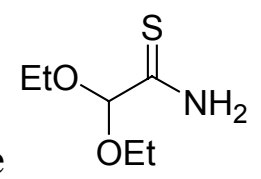

According to a modified literature procedure, ${ }^{3}$ phosphorus pentasulphide $(15.0 \mathrm{~g}, 34.0 \mathrm{mmol})$ was added to a solution of 2,2-diethoxyacetamide $(10.0 \mathrm{~g}, 68.0 \mathrm{mmol})$ in dichloromethane $(125 \mathrm{~mL})$. The mixture was stirred for $2 \mathrm{~h}$ at $0{ }^{\circ} \mathrm{C}$, the resulting dark purple mixture was filtered through Celite ${ }^{\circledR}$, washed with dichloromethane $(2 \times 10 \mathrm{~mL})$ and concentrated in vacuo. Purification by flash chromatography on silica, eluting with light petroleum-ethyl acetate $(1: 1)$, followed by recrystallisation from light petroleum-ether (7:3) gave the title compound $(2.5 \mathrm{~g}, 23 \%)$ as a brown solid, mp $93-94{ }^{\circ} \mathrm{C}$ (from light petroleum-ether) (lit. ${ }^{3} \mathrm{mp} \mathrm{81-82}{ }^{\circ} \mathrm{C}$ ): IR (nujol) 3310, 3180, 1645, 1258, 1142, 1102, 1057, 981, 886, 836, $715 \mathrm{~cm}^{-1} ;{ }^{1} \mathrm{H}$ NMR (400 MHz, $\left.\mathrm{CDCl}_{3}\right) \delta 7.95(1 \mathrm{H}, \mathrm{bs}, \mathrm{NH}), 7.90(1 \mathrm{H}, \mathrm{bs}, \mathrm{NH}), 4.98,(1$ $\mathrm{H}, \mathrm{s}, \mathrm{CH}), 3.66\left(2 \mathrm{H}, \mathrm{q}, J=7.1, \mathrm{CH}_{2} \mathrm{Me}\right), 3.59\left(2 \mathrm{H}, \mathrm{q}, J=7.1, \mathrm{CH}_{2} \mathrm{Me}\right), 1.19(6 \mathrm{H}$, app t, $J=7.1$, $\left.\mathrm{CH}_{2} \mathrm{Me}\right) ;{ }^{13} \mathrm{C}$ NMR $\left(100 \mathrm{MHz}, \mathrm{CDCl}_{3}\right) \delta 202.2(\mathrm{C}), 103.0(\mathrm{CH}), 62.9\left(\mathrm{CH}_{2}\right), 15.1(\mathrm{Me}) ; \mathrm{MS}(\mathrm{APcI}) \mathrm{m} / \mathrm{z}$ (relative intensity) $164\left(\mathrm{MH}^{+}, 7 \%\right), 118$ (100), 90 (14); Anal. Calcd for $\mathrm{C}_{6} \mathrm{H}_{13} \mathrm{NO}_{2} \mathrm{~S}: \mathrm{C}, 44.1 ; \mathrm{H}, 8.0 ; \mathrm{N}$, 8.6. Found: C, $44.1 ; \mathrm{H}, 8.0 ; \mathrm{N}, 8.6$.

\section{Ethyl 2-(diethoxymethyl)thiazole-4-carboxylate}

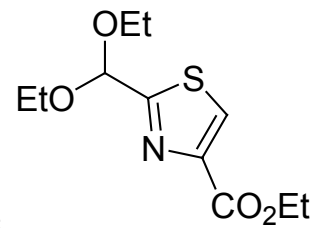

Ethyl bromopyruvate $(2.2 \mathrm{~mL}, 15.4 \mathrm{mmol})$ was added to a mixture of 2,2-diethoxythioacetamide $(2.3 \mathrm{~g}$, $14.0 \mathrm{mmol})$ and $4 \AA$ molecular sieves $(4 \mathrm{~g})$ in ethanol $(30 \mathrm{~mL})$ and the mixture heated at reflux for $1 \mathrm{~h}$. The solution was cooled, filtered through Celite $^{\circledR}$ and evaporated in vacuo. Purification by flash chromatography on silica, eluting with light petroleum-ethyl acetate (3:1), gave the title compound ${ }^{3}$ (2.5 g, 68\%) as a pale yellow oil: IR (film) 3111, 2980, 2936, 2885, 1732, 1489, 1368, 1313, 1241, 1206, 1093, 1062, 1020, $760 \mathrm{~cm}^{-1} ;{ }^{1} \mathrm{H}$ NMR $\left(400 \mathrm{MHz}, \mathrm{CDCl}_{3}\right) \delta 8.14(1 \mathrm{H}, \mathrm{s}, 5-\mathrm{H}), 5.64(1 \mathrm{H}, \mathrm{s}, \mathrm{CH})$, $4.36\left(2 \mathrm{H}, \mathrm{q}, J=7.1, \mathrm{CH}_{2} \mathrm{Me}\right), 3.69\left(2 \mathrm{H}, \mathrm{q}, J=7.1, \mathrm{OCH}_{2} \mathrm{Me}\right), 3.58\left(2 \mathrm{H}, \mathrm{q}, J=7.1, \mathrm{OCH}_{2} \mathrm{Me}\right), 1.33$ $\left(3 \mathrm{H}, \mathrm{t}, J=7.1, \mathrm{CH}_{2} \mathrm{Me}\right), 1.19\left(6 \mathrm{H}\right.$, app t, $\left.J=7.1, \mathrm{OCH}_{2} \mathrm{Me}\right) ;{ }^{13} \mathrm{C} \mathrm{NMR}\left(100 \mathrm{MHz}, \mathrm{CDCl}_{3}\right) \delta 170.1(\mathrm{C})$, $161.4(\mathrm{C}), 147.1(\mathrm{C}), 128.5(\mathrm{CH}), 98.8(\mathrm{CH}), 62.8\left(\mathrm{CH}_{2}\right), 61.5\left(\mathrm{CH}_{2}\right), 15.1(\mathrm{Me}), 14.4(\mathrm{Me}) ; \mathrm{MS}(\mathrm{APcI})$ $m / z$ (relative intensity) $261\left(\mathrm{M}^{+}, 67 \%\right), 215$ (100); HRMS calcd for $\mathrm{C}_{11} \mathrm{H}_{21} \mathrm{~N}_{2} \mathrm{O}_{4} \mathrm{~S}\left(\mathrm{MNH}_{4}\right)$ 277.1222, found 277.1218 .

\footnotetext{
${ }^{3}$ Inami, K.; Shiba, T. Bull Chem. Soc. Jpn. 1985, 58, 352.
} 


\section{Ethyl 2-formylthiazole-4-carboxylate}

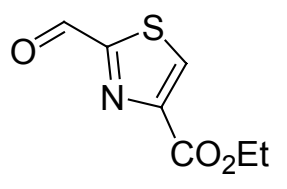

A solution of ethyl 2-(diethoxymethyl)thiazole-4-carboxylate $(2.11 \mathrm{~g}, 8.14 \mathrm{mmol})$ was heated at reflux in acetone $(175 \mathrm{~mL})$ with aqueous $2 \mathrm{~N}$ hydrochloric acid $(17 \mathrm{~mL})$ for $1 \mathrm{~h}$, cooled, evaporated in vacuo and extracted with chloroform $(100 \mathrm{~mL})$. The aqueous layer was further extracted with chloroform $(2 \mathrm{x}$ $70 \mathrm{~mL})$ and the combined organic extracts were dried $\left(\mathrm{Na}_{2} \mathrm{SO}_{4}\right)$ and concentrated in vacuo. Purification by flash chromatography on silica, eluting with light petroleum-ethyl acetate $(3: 2)$, gave the title compound (1.39 g, 92\%) as a pale yellow solid, $\mathrm{mp}$ 64.3-65.6 ${ }^{\circ} \mathrm{C}$ (from light petroleum) (lit. ${ }^{3} \mathrm{mp} \mathrm{67-68}$ ${ }^{\circ} \mathrm{C}$ ). IR (nujol) 3103, 1723, 1706, 1324, 1214, 1100, 1022, 946, 879, $767 \mathrm{~cm}^{-1}$; ${ }^{1} \mathrm{H}$ NMR (400 MHz, $\left.\mathrm{CDCl}_{3}\right) \delta 10.01(1 \mathrm{H}, \mathrm{d}, J=1.2, \mathrm{CHO}), 8.52(1 \mathrm{H}, \mathrm{d}, J=1.2,5-\mathrm{H}), 4.42\left(2 \mathrm{H}, \mathrm{q}, J=7.2, \mathrm{CH}_{2} \mathrm{Me}\right), 1.37$ (3 H, t, $\left.J=7.2, \mathrm{CH}_{2} \mathrm{Me}\right) ;{ }^{13} \mathrm{C}$ NMR $\left(100 \mathrm{MHz}, \mathrm{CDCl}_{3}\right) \delta 183.7(\mathrm{CH}), 166.1(\mathrm{C}), 160.6(\mathrm{C}), 149.5(\mathrm{C})$, $133.1(\mathrm{CH}), 62.1\left(\mathrm{CH}_{2}\right), 14.3(\mathrm{Me})$; MS (APcI) $\mathrm{m} / z$ (relative intensity) $186\left(\mathrm{MH}^{+}, 100 \%\right), 171(40)$, 158 (12), 140 (24); HRMS calcd for $\mathrm{C}_{7} \mathrm{H}_{8} \mathrm{NO}_{3} \mathrm{~S}(\mathrm{MH})$ 186.0225, found 186.0219. Anal. Calcd for $\mathrm{C}_{7} \mathrm{H}_{7} \mathrm{NO}_{3} \mathrm{~S}: \mathrm{C}, 45.5 ; \mathrm{H}, 3.8 ; \mathrm{N}, 7.6$. Found: $\mathrm{C}, 45.5 ; \mathrm{H}, 3.8 ; \mathrm{N}, 7.5$.

\section{Ethyl 2-(1-hydroxyprop-2-ynyl)thiazole-4-carboxylate}

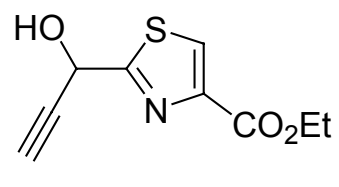

A solution of ethynyl magnesiumbromide in tetrahydrofuran $(0.5 \mathrm{M} ; 14.1 \mathrm{~mL}, 7.07 \mathrm{mmol})$ was added dropwise over $5 \mathrm{~min}$ to a solution of ethyl 2-formylthiazole-4-carboxylate $(1.31 \mathrm{~g}, 7.07 \mathrm{mmol})$ in tetrahydrofuran $(50 \mathrm{~mL})$ at $0{ }^{\circ} \mathrm{C}$ and the mixture was stirred for $30 \mathrm{~min}$ at this temperature. A further aliquot of ethynyl magnesiumbromide in tetrahydrofuran $(0.5 \mathrm{M} ; 2.8 \mathrm{~mL}, 1.41 \mathrm{mmol})$ was added and the solution stirred for a further $20 \mathrm{~min}$. Saturated aqueous ammonium chloride solution $(30 \mathrm{~mL})$ was added and the mixture was concentrated in vacuo and partitioned between water $(30 \mathrm{~mL})$ and chloroform $(60 \mathrm{~mL})$. The aqueous layer was further extracted with chloroform $(2 \times 30 \mathrm{~mL})$ and the combined organic extracts were dried $\left(\mathrm{Na}_{2} \mathrm{SO}_{4}\right)$ and concentrated in vacuo. Purification by flash chromatography on silica, eluting with light petroleum-ethyl acetate (2:3), gave the title compound (1.09 g, 73\%) as a brown oil: IR (film) 3290, 3122, 2981, 2121, 1714, 1486, 1371, 1221, 1174, 1100, 1020, 958, 922, 878, $758 \mathrm{~cm}^{-1} ;{ }^{1} \mathrm{H}$ NMR $\left(400 \mathrm{MHz}, \mathrm{CDCl}_{3}\right) \delta 8.11(1 \mathrm{H}, \mathrm{s}, 5-\mathrm{H}), 5.70,(1 \mathrm{H}, \mathrm{m}$, $\mathrm{CHOH}), 5.14,(1 \mathrm{H}, \mathrm{bm}, \mathrm{OH}), 4.30\left(2 \mathrm{H}, \mathrm{q}, J=7.2, \mathrm{CH}_{2} \mathrm{Me}\right), 2.69(1 \mathrm{H}, \mathrm{d}, J=1.9, \mathrm{CCH}), 1.29(3 \mathrm{H}, \mathrm{t}$, $\left.J=7.2, \mathrm{CH}_{2} \mathrm{Me}\right) ;{ }^{13} \mathrm{C} \mathrm{NMR}\left(100 \mathrm{MHz}, \mathrm{CDCl}_{3}\right) \delta 172.3(\mathrm{C}), 161.3(\mathrm{C}), 146.6(\mathrm{C}), 128.6(\mathrm{CH}), 81.3(\mathrm{C})$, $75.5(\mathrm{CH}), 61.7\left(\mathrm{CH}_{2}\right), 61.3(\mathrm{CH}), 14.3(\mathrm{Me})$; MS (EI) m/z (relative intensity) $211\left(\mathrm{M}^{+}, 20 \%\right), 182(74)$, 
166 (58), 158 (22), 139 (51), 121 (26), 112 (82), 84 (34), 55 (100), 45 (58); HRMS calcd for $\mathrm{C}_{9} \mathrm{H}_{10} \mathrm{NO}_{3} \mathrm{~S}(\mathrm{MH}) 212.0381$, found 212.0380.

\section{Ethyl 2-(propynoyl)thiazole-4-carboxylate (10)}

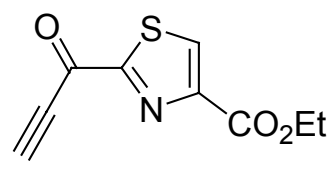

Activated manganese(IV) oxide (69 $\mathrm{mg}, 0.71 \mathrm{mmol})$ was added to a solution of ethyl 2-(1hydroxyprop-2-ynyl)thiazole-4-carboxylate $(28 \mathrm{mg}, 0.14 \mathrm{mmol})$ in dichloromethane $(2 \mathrm{~mL})$. After stirring for $10 \mathrm{~min}$ at room temperature, a further aliquot of activated manganese(IV) oxide (69 mg, $0.71 \mathrm{mmol}$ ) was added and the mixture was stirred for a further $10 \mathrm{~min}$. The resulting mixture was filtered through Celite ${ }^{\circledR}$, washed with dichloromethane $(1 \mathrm{~mL})$ and evaporated without heat under a nitrogen atmosphere to give the title compound $(28 \mathrm{mg}, 100 \%)$ as a pale yellow solid that was used without further purification, mp 101-102 ${ }^{\circ} \mathrm{C}$ (from dichloromethane): IR $\left(\mathrm{CH}_{2} \mathrm{Cl}_{2}\right)$ 3054, 2984, 2103 , 1732, 1650, 1447, 1367, 1334, 1222, 1104, 1020, 969, $932 \mathrm{~cm}^{-1} ;{ }^{1} \mathrm{H}$ NMR (400 MHz, $\left.\mathrm{CDCl}_{3}\right) \delta 8.44(1$ $\mathrm{H}, \mathrm{s}, 5-\mathrm{H}), 4.40\left(2 \mathrm{H}, \mathrm{q}, J 7.1 \mathrm{CH}_{2} \mathrm{Me}\right), 3.66(1 \mathrm{H}, \mathrm{s}, \mathrm{CCH}), 1.40\left(3 \mathrm{H}, \mathrm{t}, J 7.1, \mathrm{CH}_{2} \mathrm{Me}\right) ;{ }^{13} \mathrm{C} \mathrm{NMR}(100$ $\left.\mathrm{MHz}, \mathrm{CDCl}_{3}\right) \delta 169.5(\mathrm{C}), 166.5(\mathrm{C}), 161.0(\mathrm{C}), 149.0(\mathrm{C}), 134.0(\mathrm{CH}), 85.4(\mathrm{CH}), 79.3(\mathrm{C}), 62.5$ $\left(\mathrm{CH}_{2}\right), 14.7$ (Me); MS (APcI) m/z (relative intensity) $210\left(\mathrm{MH}^{+}, 100 \%\right)$; HRMS calcd for $\mathrm{C}_{9} \mathrm{H}_{8} \mathrm{NO}_{3} \mathrm{~S}$ (MH) 210.0225, found 210.0224.

\section{$(R, 4 E)$-Ethyl 2-[5-amino-6-( $\mathrm{N}$-tert-butoxycarbonyl)amino-4-} (ethoxycarbonyl)hepta-2,4-dienoyl]-thiazole-4-carboxylate (11)

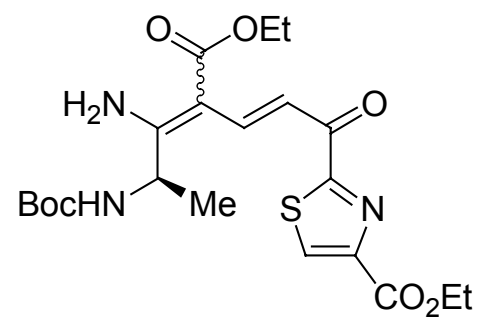

To a solution of ethyl 2-(propynoyl)thiazole-4-carboxylate (10) $(29 \mathrm{mg}, 0.14 \mathrm{mmol})$ in ethanol $(5 \mathrm{~mL})$ was added (R)-ethyl 3-amino-4-( $N$-tert-butoxycarbonyl)aminopent-2-enoate (6) (41 mg, $0.16 \mathrm{mmol}$ ). The reaction mixture was stirred at $50{ }^{\circ} \mathrm{C}$ for $10 \mathrm{~min}$ and concentrated in vacuo. Purification by flash chromatography on silica, eluting with light petroleum-ethyl acetate (1:1), gave the title compound (53 $\mathrm{mg}, 82 \%$ ) as a yellow solid, $\mathrm{mp} 176-178^{\circ} \mathrm{C}$ (from methanol): $[\alpha]_{\mathrm{D}}{ }^{25}-153.3$ (c 1.25, $\mathrm{CHCl}_{3}$ ); $\mathrm{IR}(\mathrm{KBr})$ 3424, 2976, 1720, 1654, 1559, 1509, 1458, 1368, 1328, 1278, 1222, $1022 \mathrm{~cm}^{-1}$; ${ }^{1} \mathrm{H}$ NMR $(400 \mathrm{MHz}$, $d_{6}$-DMSO) $\delta 9.77(1 \mathrm{H}$, br s, NHH), 8.82 (1 H, s, SCH), $8.25(1 \mathrm{H}, \mathrm{d}, J=15.0,3-\mathrm{H}), 8.18(1 \mathrm{H}$, br s, $\mathrm{NH} H), 7.59(1 \mathrm{H}, \mathrm{d}, J=15.0,2-\mathrm{H}), 7.38(1 \mathrm{H}, \mathrm{d}, J=5.6, \mathrm{NHBoc}), 4.96(1 \mathrm{H}, \mathrm{m}, \mathrm{CHMe}), 4.34(2 \mathrm{H}, \mathrm{q}$, $\left.J=7.1, \mathrm{OCH}_{2} \mathrm{Me}\right), 4.25\left(2 \mathrm{H}, \mathrm{q}, J=7.1, \mathrm{OCH}_{2} \mathrm{Me}\right), 1.40\left(3 \mathrm{H}, \mathrm{t}, J=7.1, \mathrm{OCH}_{2} \mathrm{Me}\right), 1.39(9 \mathrm{H}, \mathrm{s}$, $\mathrm{CMe}_{3}$ ), $1.33\left(3 \mathrm{H}, \mathrm{t}, J=7.1, \mathrm{OCH}_{2} \mathrm{Me}\right), 1.33$ (3 H, d, $\left.J=6.7, \mathrm{CHMe}\right) ;{ }^{13} \mathrm{C} \mathrm{NMR}\left(100 \mathrm{MHz}, d_{6}\right.$-DMSO) $\delta 177.6(\mathrm{C}), 172.0(\mathrm{C}), 167.9(\mathrm{C}), 166.4(\mathrm{C}), 158.3(\mathrm{C}), 152.4(\mathrm{C}), 144.9(\mathrm{C}), 139.6(\mathrm{CH}), 131.8(\mathrm{CH})$, 
109.6 (CH), $90.3(\mathrm{C}), 76.6(\mathrm{C}), 58.7\left(\mathrm{CH}_{2}\right), 57.5\left(\mathrm{CH}_{2}\right), 44.9(\mathrm{CH}), 25.8(\mathrm{Me}), 17.5(\mathrm{Me}), 11.8(\mathrm{Me})$, 11.7 (Me); MS (APcI) $m / z$ (relative intensity) $468\left(\mathrm{MH}^{+}, 100 \%\right.$ ); HRMS calcd for $\mathrm{C}_{21} \mathrm{H}_{30} \mathrm{~N}_{3} \mathrm{O}_{7} \mathrm{~S}(\mathrm{MH})$ 468.1799, found 468.1796.

\section{(R)-Ethyl 6-[4-(ethoxycarbonyl)thiazol-2-yl]-2-[1-(N-tert-}

\section{butoxycarbonyl)aminoethyl]pyridine-3-carboxylate (8a)}

A solution of (R)-ethyl 4-(N-tert-butoxycarbonyl)amino-3-

oxopentanoate (9) $(107 \mathrm{mg}, 0.41 \mathrm{mmol})$ and ammonium acetate (158

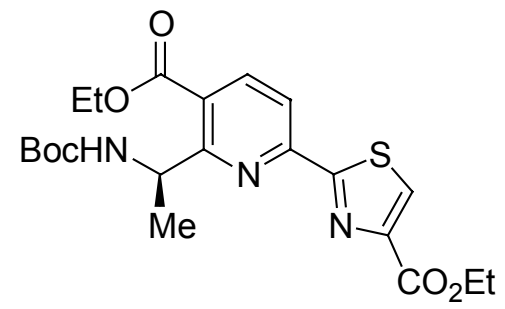

$\mathrm{mg}, 2.0 \mathrm{mmol})$ in ethanol $(10 \mathrm{~mL})$ was stirred at room temperature for $4 \mathrm{~h}$ then a solution of ethyl 2(propynoyl)thiazole-4-carboxylate $(\mathbf{1 0})(69 \mathrm{mg}, 0.33 \mathrm{mmol})$ in ethanol $(5 \mathrm{~mL})$ was added. The reaction mixture was stirred at room temperature for $1 \mathrm{~h}$ and cooled to $0{ }^{\circ} \mathrm{C}$. $\mathrm{N}$-Iodosuccinimide $(92 \mathrm{mg}, 0.41$ mmol) was added portionwise, the mixture was stirred at $0{ }^{\circ} \mathrm{C}$ for $15 \mathrm{~min}$ and then concentrated. The residue was partitioned between ethyl acetate $(15 \mathrm{~mL})$ and saturated aqueous sodium hydrogen carbonate solution $(15 \mathrm{~mL})$. The aqueous layer was further extracted with ethyl acetate $(10 \mathrm{~mL})$ and the organic layers were combined, washed sequentially with saturated aqueous sodium hydrogen carbonate solution $(10 \mathrm{~mL})$, and brine $(10 \mathrm{~mL})$, dried $\left(\mathrm{Na}_{2} \mathrm{SO}_{4}\right)$, evaporated in vacuo, and purified by flash chromatography on silica, eluting with light petroleum-ethyl acetate (7:3), to give the title compound ${ }^{4}$ (82 mg, 55\%) as a pale yellow oil: $[\alpha]_{\mathrm{D}}^{21}+39.0\left(c\right.$ 1.06, $\left.\mathrm{CHCl}_{3}\right)$; IR (KBr) 3361, 2983, 2933, 1738, 1723, 1689, 1582, 1528, 1449, 1366, 1336, 1270, 1208, 1174, 1047, 1029, 855, 806, $756 \mathrm{~cm}^{-1} ;{ }^{1} \mathrm{H}$ NMR (400 MHz, $d_{6}$-acetone) $\delta 8.46(1 \mathrm{H}, \mathrm{s}, \mathrm{SCH}), 8.31(1 \mathrm{H}, \mathrm{d}, J=8.1, \mathrm{PyH}), 8.08(1 \mathrm{H}, \mathrm{d}, J=8.1$, PyH), 6.26 (1 H, d, $J=8.2, \mathrm{NH}), 5.58(1 \mathrm{H}, \mathrm{m}, \mathrm{CHMe}), 4.31\left(2 \mathrm{H}, \mathrm{q}, J=7.2, \mathrm{OCH}_{2} \mathrm{Me}\right), 4.26(2 \mathrm{H}, \mathrm{q}, J$ $\left.=7.1, \mathrm{OCH}_{2} \mathrm{Me}\right), 1.37(3 \mathrm{H}, \mathrm{d}, J=6.8, \mathrm{CHMe}), 1.30\left(3 \mathrm{H}, \mathrm{t}, J=7.1, \mathrm{OCH}_{2} \mathrm{Me}\right), 1.26(3 \mathrm{H}, \mathrm{t}, J=7.2$, $\mathrm{OCH}_{2} \mathrm{Me}$ ), $1.24\left(9 \mathrm{H}, \mathrm{s}, \mathrm{CMe}_{3}\right) ;{ }^{13} \mathrm{C}$ NMR (100 MHz, $\left.\mathrm{CDCl}_{3}\right) \delta 168.7(\mathrm{C}), 165.2(\mathrm{C}), 162.9(\mathrm{C}), 161.3$ (C), 155.2 (C), 151.7 (C), 148.7 (C), 140.3 (CH), 130.3 (CH), 125.2 (C), 118.1 (CH), 79.3 (C), 61.9 $\left(\mathrm{CH}_{2}\right), 61.7\left(\mathrm{CH}_{2}\right), 48.7(\mathrm{CH}), 28.4(\mathrm{Me}), 22.9(\mathrm{Me}), 14.4(\mathrm{Me}), 14.2(\mathrm{Me})$; MS (APcI) $m / z$ (relative intensity) 450 ( $\mathrm{MH}^{+}, 100 \%$ ), 394 (23), 350 (9); HRMS calcd for $\mathrm{C}_{21} \mathrm{H}_{28} \mathrm{~N}_{3} \mathrm{O}_{6} \mathrm{~S}(\mathrm{MH}) 450.1693$, found 450.1691 .

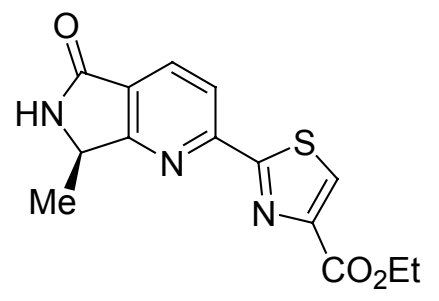

\footnotetext{
${ }^{4}$ HPLC: ee $=96 \%$; Chiralpak AD, hexane-propan-2-ol $(95: 5), \lambda_{\max }=311 \mathrm{~nm}, 1.0 \mathrm{~mL} \mathrm{~min}{ }^{-1}, R_{\mathrm{T}}=14.8 \mathrm{~min}(S$-isomer has $\left.R_{\mathrm{T}}=12.4 \mathrm{~min}\right)$.
} 


\section{(R)-2-[4-(Ethoxycarbonyl)thiazol-2-yl]-7-methyl-5-oxo-6,7-dihydro-5H-pyrrolo[3,4-b]pyridine (12)}

A solution of $(R)$-ethyl 6-[4-(ethoxycarbonyl)thiazol-2-yl]-2-[1-(N-tert-butoxycarbonyl)aminoethyl]pyridine-3-carboxylate $(\mathbf{8 a})(93 \mathrm{mg}, 0.21 \mathrm{mmol})$ in aqueous hydrochloric acid $(6 \mathrm{~N} ; 10 \mathrm{~mL})$ was stirred at room temperature for $6 \mathrm{~h}$, neutralised to $\mathrm{pH} 7$ with aqueous sodium hydroxide $(5 \mathrm{~N})$ and extracted with dichloromethane $(3 \times 20 \mathrm{~mL})$. The organic layers were combined, dried $\left(\mathrm{Na}_{2} \mathrm{SO}_{4}\right)$ and evaporated in vacuo. Purification by flash chromatography on silica, eluting with ethyl acetate, gave the title compound $^{5}$ (56 mg, 89\%) as a colorless solid, mp $236-238{ }^{\circ} \mathrm{C}$ (from methanol): $[\alpha]_{\mathrm{D}}{ }^{24}+48.1(c 0.54$, $\mathrm{CHCl}_{3}$ ); IR (KBr) 3200, 2926, 1700, 1600, 1412, 1247, 1098, 1022, $805 \mathrm{~cm}^{-1} ;{ }^{1} \mathrm{H}$ NMR (400 MHz, $d_{6^{-}}$ DMSO) $\delta 9.14(1 \mathrm{H}, \mathrm{s}, \mathrm{NH}), 8.79(1 \mathrm{H}, \mathrm{s}, \mathrm{SCH}), 8.33$ (1 H, d, J= 8.0, PyH), 8.30 (1 H, d, J= 8.0, PyH), $4.83(1 \mathrm{H}, \mathrm{q}, J=6.8, \mathrm{CHMe}), 4.42\left(2 \mathrm{H}, \mathrm{q}, J=7.1, \mathrm{OCH}_{2} \mathrm{Me}\right), 1.52(3 \mathrm{H}, \mathrm{d}, J=6.8, \mathrm{CHMe}), 1.41(3 \mathrm{H}$, $\left.\mathrm{t}, J=7.1, \mathrm{OCH}_{2} \mathrm{Me}\right) ;{ }^{13} \mathrm{C} \mathrm{NMR}\left(100 \mathrm{MHz}, d_{6}\right.$-DMSO) $\delta 168.9$ (C), 168.5 (C), 166.8 (C), 161.0 (C), $152.5(\mathrm{C}), 148.0(\mathrm{C}), 133.9(\mathrm{CH}), 132.6(\mathrm{CH}), 127.3(\mathrm{C}), 119.7(\mathrm{CH}), 61.5\left(\mathrm{CH}_{2}\right), 53.5(\mathrm{CH}), 19.0$ (Me), 14.7 (Me); MS (APcI) m/z (relative intensity) $304\left(\mathrm{MH}^{+}, 100 \%\right.$ ); HRMS calcd for $\mathrm{C}_{14} \mathrm{H}_{14} \mathrm{~N}_{3} \mathrm{O}_{3} \mathrm{~S}$ (MH) 304.0750, found 304.0750.<smiles>C[C@@H]1NC(=O)c2ccc(-c3nc(C(=O)O)cs3)nc21</smiles>

\section{(R)-2-(4-Carboxythiazol-2-yl)-7-methyl-5-oxo-6,7-dihydro-5H-pyrrolo[3,4-b]pyridinium chloride}

Lithium hydroxide monohydrate $(25 \mathrm{mg}, 0.60 \mathrm{mmol})$ was added to a solution of (R)-2-[4(ethoxycarbonyl)thiazol-2-yl]-7-methyl-5-oxo-6,7-dihydro-5H-pyrrolo[3,4-b]pyridine (12) (92 mg, $0.30 \mathrm{mmol})$ in tetrahydrofuran-water $(1: 1)(30 \mathrm{~mL})$ at room temperature. The solution was stirred for 3 $\mathrm{h}$, evaporated in vacuo, and partitioned between water $(30 \mathrm{~mL})$ and dichloromethane $(30 \mathrm{~mL})$. The aqueous layer was separated, acidified to pH 6 with aqueous hydrochloric acid $(3 \mathrm{~N})$ and concentrated. The precipitate was filtered, washed sequentially with water $(2 \mathrm{~mL})$ and acetone $(5 \mathrm{~mL})$, and dried in a vacuum dessicator for $24 \mathrm{~h}$ to give the title compound (72 $\mathrm{mg}, 77 \%$ ) as a colorless solid that was used without further purification, $\mathrm{mp} 287^{\circ} \mathrm{C} \mathrm{dec} \mathrm{(from} \mathrm{aqueous} \mathrm{methanol):}[\alpha]_{\mathrm{D}}{ }^{30}+30.8(c 0.26, \mathrm{MeOH})$; UV $\lambda_{\max }$ (methanol) $234 \mathrm{~nm}(\varepsilon$ 12400), $314 \mathrm{~nm}(\varepsilon$ 17000); IR (KBr) 3234, 2925, 2517, 1890, 1716,

${ }^{5}$ HPLC: ee $=94 \%$; Chiralcel OD, hexane-propan-2-ol (90:10), $\lambda_{\max }=314 \mathrm{~nm}, 1.0 \mathrm{~mL} \mathrm{~min}^{-1}, R_{\mathrm{T}}=52.6 \min (S$-isomer has $\left.R_{\mathrm{T}}=34.8 \mathrm{~min}\right)$. 
1648, 1601, 1412, 1364, 1221, 1099, 1013, 744, $725 \mathrm{~cm}^{-1} ;{ }^{1} \mathrm{H}$ NMR (400 MHz, $d_{6}$-DMSO) $\delta 9.14(1 \mathrm{H}$, s, NH), 8.69 (1 H, s, SCH), 8.34 (1 H, d, $J=8.0$, PyH), $8.31(1 \mathrm{H}, \mathrm{d}, J=8.0$, PyH), 4.84 (1 H, q, $J=$ 6.8, CHMe), 1.52 (3 H, d, $J=6.8, \mathrm{CHMe}) ;{ }^{13} \mathrm{C}$ NMR (100 MHz, $d_{6}$-DMSO) $\delta 168.4(\mathrm{C}), 167.6$ (C), $166.3(\mathrm{C}), 161.9(\mathrm{C}), 152.2(\mathrm{C}), 148.7$ (C), $133.3(\mathrm{CH}), 131.6(\mathrm{CH}), 126.7(\mathrm{C}), 119.2(\mathrm{CH}), 53.0(\mathrm{CH})$, $18.5(\mathrm{Me})$; MS (ES) $m / z$ (relative intensity) $298\left(\mathrm{M}-\mathrm{HCl}+\mathrm{Na}^{+}, 100 \%\right), 276\left(\mathrm{M}-\mathrm{HCl}+\mathrm{H}^{+}, 53\right)$; HRMS calcd for $\mathrm{C}_{12} \mathrm{H}_{10} \mathrm{~N}_{3} \mathrm{O}_{3} \mathrm{~S}(\mathrm{M}-\mathrm{HCl}+\mathrm{H})$ 276.0437, found 276.0441.<smiles>C[C@@H]1NC(=O)c2ccc(-c3nc(C(=O)O)cs3)nc21</smiles>

\section{(R)-2-(4-Carboxythiazol-2-yl)-7-methyl-5-oxo-6,7-dihydro-5 $H$-pyrrolo[3,4-b]pyridine (2)}

To a stirred solution of (R)-2-(4-carboxythiazol-2-yl)-7-methyl-5-oxo-6,7-dihydro-5H-pyrrolo[3,4b]pyridinium chloride (13) $(25 \mathrm{mg}, 0.08 \mathrm{mmol})$ in dry methanol $(50 \mathrm{~mL})$ was added dry polymerbound 4-( $N$-benzyl- $N$-methylamino)pyridine (32 mg, $0.08 \mathrm{mmol})$ and powdered $4 \AA$ molecular sieves $(2$ g). The reaction mixture was stirred at room temperature for $18 \mathrm{~h}$, filtered through Celite ${ }^{\circledR}$, washed with methanol $(2 \times 20 \mathrm{~mL})$ and concentrated in vacuo. Recrystallisation from methanol gave the title compound $^{6,7}(18 \mathrm{mg}, 80 \%)$ as a colorless solid, mp $185{ }^{\circ} \mathrm{C} \mathrm{dec} \mathrm{(from} \mathrm{methanol):}[\alpha]_{\mathrm{D}}{ }^{28}+22.7(c 0.12$,

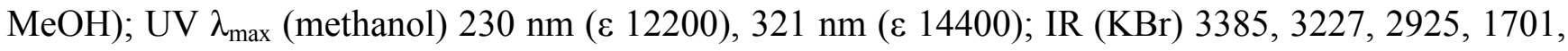
$1601,1485,1413,1372,1289,1017,858,787,756,724 \mathrm{~cm}^{-1} ;{ }^{1} \mathrm{H}$ NMR (400 MHz, $d_{6}$-DMSO) $\delta 9.05$ (1 H, s, NH), 8.38 (1 H, d, $J=8.0, \mathrm{PyH}), 8.25$ (1 H, d, J=8.0, PyH), 8.01 (1 H, s, SCH), 4.80 (1 H, q, $J=6.6, \mathrm{CHMe}), 1.51(3 \mathrm{H}, \mathrm{d}, J=6.6, \mathrm{CHMe}) ;{ }^{13} \mathrm{C} \mathrm{NMR}\left(100 \mathrm{MHz}, d_{6}\right.$-DMSO) $\delta 168.6(\mathrm{C}), 167.1(\mathrm{C})$, $165.9(\mathrm{C}), 164.6(\mathrm{C}), 160.4(\mathrm{C}), 153.7(\mathrm{C}), 133.3(\mathrm{CH}), 126.4(\mathrm{C}), 125.1(\mathrm{CH}), 119.7(\mathrm{CH}), 53.5(\mathrm{CH})$, 19.1 (Me); MS (ES) $m / z$ (relative intensity) $320\left(\mathrm{M}-\mathrm{H}+2 \mathrm{Na}^{+}, 100 \%\right), 298\left(\mathrm{M}+\mathrm{Na}^{+}, 18\right), 232$ (32), 109 (28); HRMS calcd for $\mathrm{C}_{12} \mathrm{H}_{8} \mathrm{~N}_{3} \mathrm{O}_{3} \mathrm{~S}(\mathrm{M}-\mathrm{H})$ 274.0292, found 274.0290.

\footnotetext{
${ }^{6}$ HPLC: ee $=88 \%$; Chiralpak OD-R, water-acetonitrile (95:5), $\lambda_{\max }=321 \mathrm{~nm}, 0.5 \mathrm{~mL} \mathrm{~min}^{-1}, R_{\mathrm{T}}=18.9 \min (S$-isomer has $\left.R_{\mathrm{T}}=15.0 \mathrm{~min}\right)$.

${ }^{7}$ Literature reported data (ref 6 and 20 in article): FAB mass, positive $276(\mathrm{M}+\mathrm{H})+$, negative $274(\mathrm{M}-\mathrm{H}) ;{ }^{-} \mathrm{H}-\mathrm{NMR}(\delta \mathrm{ppm}$ DMSO- $\left.d_{6}\right) 1.45(3 \mathrm{H}, \mathrm{d}), 4.74(1 \mathrm{H}, \mathrm{q}), 8.01(1 \mathrm{H}, \mathrm{s}), 8.18(1 \mathrm{H}, \mathrm{d}, J=8 \mathrm{~Hz}), 8.38(1 \mathrm{H}, \mathrm{d}, J=8 \mathrm{~Hz}), 9.00\left(1 \mathrm{H}, \mathrm{s}, \mathrm{D}_{2} \mathrm{O}\right.$ exchangeable); ${ }^{13} \mathrm{C}-\mathrm{NMR} 168.2,166.5,165.6,164.3,159.8,153.2,132.7,126.9,124.6,119.3,53.0,18.6$; UV $\lambda_{\max }$ (methanol) $230 \mathrm{~nm}(\varepsilon$ 12200), $321 \mathrm{~nm}(\varepsilon$ 14400).
} 


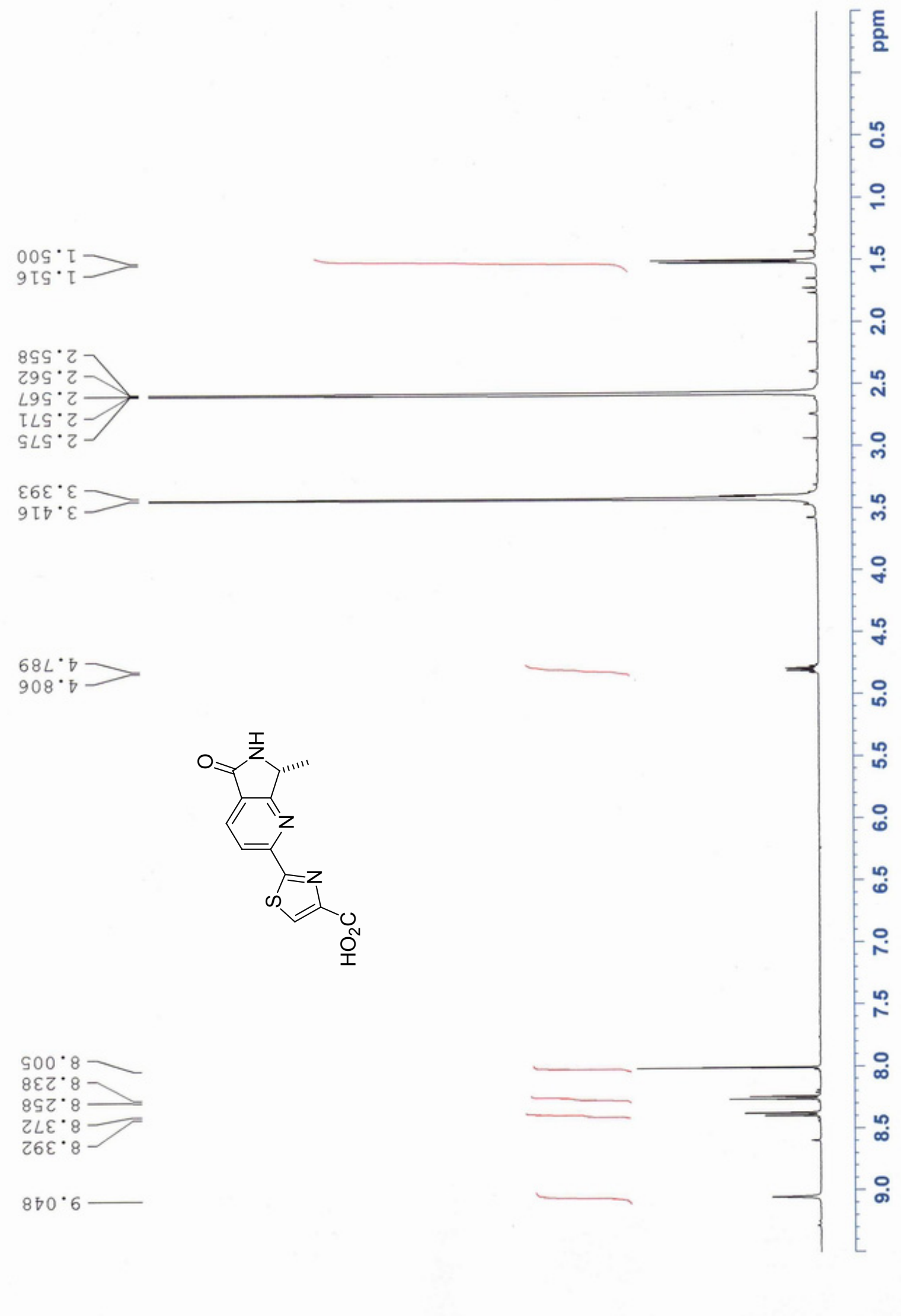


$90 \tau^{*} 6 \tau$

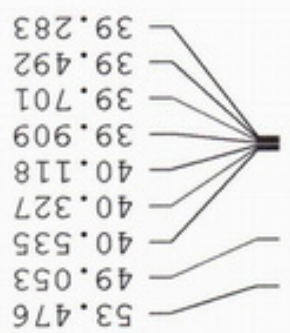

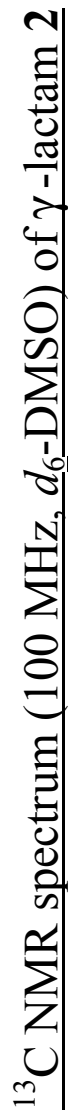

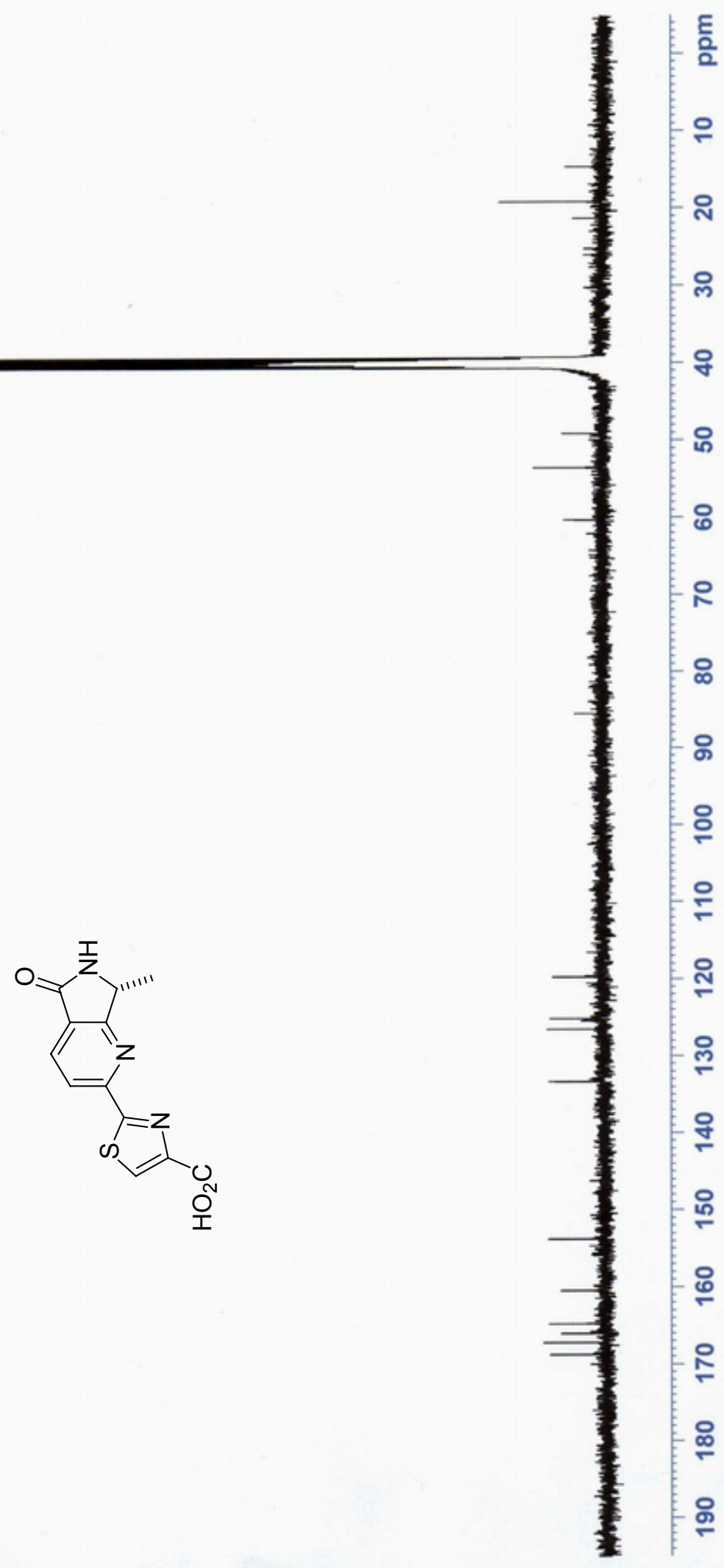

